\title{
Cosmological expansion in the Randall-Sundrum brane world scenario
}

\author{
Éanna É. Flanagan,* S.-H. Henry Tye, ${ }^{\dagger}$ and Ira Wasserman ${ }^{\ddagger}$ \\ Laboratory for Nuclear Studies and Center for Radiophysics and Space Research, Cornell University, Ithaca, New York 14853
}

(Received 29 October 1999; published 25 July 2000)

\begin{abstract}
The cosmology of the Randall-Sundrum scenario for a positive tension brane in a 5D universe with localized gravity has been studied previously. In the radiation-dominated universe, it was suggested that there are two solutions for the cosmic scale factor $a(t)$ : the standard solution $a \sim t^{1 / 2}$, and a solution $a \sim t^{1 / 4}$, which is incompatible with standard big bang nucleosynthesis. In this paper, we reconsider expansion of the Universe in this scenario. We derive and solve a first order, linear differential equation for $H^{2}$, the square of the expansion rate of the Universe, as a function of $a$. The differences between our equation for $H^{2}$ and the relationship found in standard cosmology are (i) there is a term proportional to density squared (a fact already known), which is small when the density is small compared to the brane tension, and (ii) there is a contribution which acts like a relativistic fluid. We show that this second contribution is due to gravitational degrees of freedom in the bulk. Thus, we find that there need not be any conflict between cosmology of the Randall-Sundrum scenario and the standard model of cosmology. We discuss how reheating at the end of inflation leads to the correct relationship between matter density and expansion rate, $H^{2} \rightarrow 8 \pi G \rho_{m} / 3$, and the conditions that must be met for the expansion rate of the universe to be close to its standard model value around the epoch of cosmological nucleosynthesis.
\end{abstract}

PACS number(s): 04.50.+h, 11.25.Mj, 98.80.Cq

\section{INTRODUCTION}

Recently Randall and Sundrum [1] presented a new static solution to the 5D (classical) Einstein equations in which spacetime is flat on a 3-brane with positive tension provided that the bulk has an appropriate negative cosmological constant. Even if the fifth dimension is uncompactified, standard 4D gravity (specifically, Newton's force law) is reproduced on the brane. In contrast with the compactified case [2], this follows because the near-brane geometry traps the massless graviton.

To see if such a scenario is viable phenomenologically, one application to check is the evolution of the early Universe $[3,4]$. It was pointed out [5] that a $5 \mathrm{D}$ universe with branes may have a rather unconventional, and perhaps unacceptable, cosmology. The inclusion of matter inside the brane is suggested naturally by the brane world picture. So it is important to see if the standard expanding Universe can be recovered by extending the static solution to a timedependent one when matter or radiation inside the brane is included. As shown in Ref. [6], the standard matterdominated expanding Universe is recovered for large enough brane tension. However, Ref. [6] proposed two possible behaviors for the cosmic scale factor, $a(t)$, during the radiation-dominated universe: (i) $a(t) \sim t^{1 / 4}$ (first found in Ref. [5]), or (ii) $a(t) \sim t^{1 / 2}$ (found in Ref. [6]). That there might be two different powerlaw solutions is not surprising, since a non-linear second order differential equation may have more than one such solution, and initial conditions will determine the precise time evolution of $a(t)$ (which may not be exactly a power law, but might be close to either of the candidate power laws at different times). On the other hand,

\footnotetext{
*Email address: eef3@cornell.edu

†Email address: tye@mail.lns.cornell.edu

‡Email address: ira@spacenet.tn.cornell.edu
}

to match the known observations of the expanding Universe, at least back to the time of electron-positron annihilation and nucleosynthesis, the expansion rate of the Universe should be approximately $H^{2}=8 \pi G \rho / 3$, its value in standard big bang cosmology. Actually this requirement is stronger than demanding $a(t) \sim t^{1 / 2}$ : agreement with the well-established picture of light element synthesis in the early universe [7] constrains $H$ as a function of temperature $T$ for $T$ $\sim 100 \mathrm{keV}-\mathrm{a}$ few MeV.

In this paper, we clarify the situation with respect to the cosmology of the Randall-Sundrum scenario. First, we elucidate why the evolution equation that one obtains for the scale factor [Eq. (24) below] is second order in time rather than first order in time as in standard cosmology. The reason is that a piece of the gravitational dynamics in the bulk is coupled to the brane dynamics, and thus there is an extra free parameter in the cosmological equations, describing the amount of effective 4D energy density due to the 5D gravitational degrees of freedom. The most general bulk metric compatible with homogeneity and isotropy is a black hole anti-de Sitter metric [8], and the free parameter is determined by mass parameter of this bulk solution. This conclusion has also been reached by Kraus [9].

Second, we derive and solve a first-order differential equation for $H^{2}$ as a function of $\log a$. This equation shows that the effect of bulk gravitational degrees of freedom on the 4D dynamics is to add a new term that decays with expansion just like a relativistic fluid (but which may be positive or negative). This new contribution to $H^{2}$ is degenerate with the contributions from relativistic particles such as photons and neutrinos in the early universe. Thus, if significant, it would alter the relationship between $H$ and temperature $T$ of the early universe, even though it would still allow $a(t) \sim t^{1 / 2}$. Since it is the dependence of $H$ on $T$ that is constrained by comparing observed light element abundances with the theory of cosmological nucleosynthesis $[7,10]$, the new terms 
due to bulk gravitational degrees of freedom must be small enough to preserve the concordance between theory and observation. We show that if we start with an inflationary epoch $[11,3,12]$, and follow the universe as it evolves into the radiation-dominated phase, it is possible (even likely) that the new term becomes insignificant by the time the universe reheats at the end of inflation. Our final result for $\mathrm{H}^{2}$ shows that standard cosmology can be recovered at late times in the Randall-Sundrum scenario in both the radiation and matter dominated eras.

\section{DERIVATION OF THE BRANE DYNAMICS}

In this section we derive the equations governing the dynamics of matter and geometry on the brane, using a local expansion of the metric near the brane. We consider a 5D spacetime with coordinates $x^{A}=\left(x^{0}, x^{1}, x^{2}, x^{3}, x^{4}\right)$ $=\left(t, x^{1}, x^{2}, x^{3}, y\right)$, and we assume that there is a single brane located at $y=0$. We assume the following form for the $5 \mathrm{D}$ metric: ${ }^{1}$

$d s^{2}=\exp [2 \beta(y, t)]\left(-d t^{2}+d y^{2}\right)+\exp [2 \alpha(y, t)] \delta_{i j} d x^{i} d x^{j}$.

Thus, the metric only depends on $t$ and $y$, and is flat in ordinary 3D space (labeled by Latin indices which run over $1,2,3)$. For simplicity, we also restrict attention to the case where $\alpha$ and $\beta$ are even functions of $y$. For this metric the non-zero components of the Einstein tensor, as shown by Binétruy et al. [5], are

$$
\begin{aligned}
& G_{00}=3\left[\dot{\alpha}^{2}+\dot{\alpha} \dot{\beta}-\alpha^{\prime \prime}-2 \alpha^{\prime 2}+\alpha^{\prime} \beta^{\prime}\right], \\
& G_{i j}=\delta_{i j} e^{2(\alpha-\beta)}\left[-2 \ddot{\alpha}-3 \dot{\alpha}^{2}-\ddot{\beta}+2 \alpha^{\prime \prime}+3 \alpha^{\prime 2}+\beta^{\prime \prime}\right], \\
& G_{44}=G_{y y}=3\left[-\ddot{\alpha}-2 \dot{\alpha}^{2}+\dot{\alpha} \dot{\beta}+\alpha^{\prime 2}+\alpha^{\prime} \beta^{\prime}\right],
\end{aligned}
$$

and

$$
G_{04}=3\left[\beta^{\prime} \dot{\alpha}+\alpha^{\prime} \dot{\beta}-\dot{\alpha}^{\prime}-\dot{\alpha} \alpha^{\prime}\right],
$$

where dots denote derivatives with respect to $t$ and primes with respect to $y$.

We assume that $\alpha$ and $\beta$ are smooth functions of $|y|$ and of $t$, i.e., $\alpha(y, t)=\hat{\alpha}(|y|, t)$ and $\beta(y, t)=\hat{\beta}(|y|, t)$, where the functions $\hat{\alpha}(\xi, t)$ and $\hat{\beta}(\xi, t)$ are smooth in a neighborhood of $\xi=0$. Then the derivative $\partial \alpha / \partial y$ will generically be discontinuous across the brane, as in Ref. [1]. We define

$$
\alpha_{1}(t)=\lim _{y \rightarrow 0^{+}} \frac{\partial \alpha(y, t)}{\partial y}=\left.\frac{\partial \hat{\alpha}(\xi, t)}{\partial \xi}\right|_{\xi=0}
$$

\footnotetext{
${ }^{1}$ Binétruy et al. [5] used the metric

$$
d s^{2}=-n^{2}(y, t) d t^{2}+b^{2}(y, t) d y^{2}+a^{2}(y, t) \delta_{i j} d x^{i} d x^{j},
$$
}

but did not employ the freedom to redefine the coordinates $y$ and $t$ to put the metric in the simpler form (1) used here. and

$$
\beta_{1}(t)=\lim _{y \rightarrow 0^{+}} \frac{\partial \beta(y, t)}{\partial y}=\left.\frac{\partial \hat{\beta}(\xi, t)}{\partial \xi}\right|_{\xi=0} .
$$

It follows that

$$
\alpha_{, y y}(y, t)=\hat{\alpha}_{, \xi \xi}(|y|, t)+2 \alpha_{1}(t) \delta(y),
$$

together with a similar equation for $\beta_{, y y}$.

We now substitute the relation (8) into the Einstein tensor components (2)-(5), and insert into the 5D Einstein equations $G_{A B}=\kappa^{2} T_{A B}$ where the energy momentum tensor ${ }^{2}$ is

$$
\begin{aligned}
T_{B}^{A}= & \operatorname{diag}[(-\rho, p, p, p, 0) \delta(y) \exp (-\beta) \\
& \left.+\left(\Lambda_{b}, \Lambda_{b}, \Lambda_{b}, \Lambda_{b}, \Lambda_{b}\right)\right] .
\end{aligned}
$$

Here $\rho$ and $p$ are the density and pressure of the matter on the brane, and $\Lambda_{b}>0$ is proportional to minus the bulk cosmological constant. ${ }^{3}$ The result is, first, two equations obtained by equating the coefficients of the $\delta(y)$ in the $G_{00}$ and $G_{i j}$ equations:

$$
\begin{aligned}
& \alpha_{1}=-\frac{\kappa^{2} \rho}{6} \exp [\beta(0, t)], \\
& \beta_{1}=\frac{\kappa^{2}(2 \rho+3 p)}{6} \exp [\beta(0, t)] .
\end{aligned}
$$

Second, there are the smooth pieces of the equations. Since we can restrict attention to $y>0$ for these pieces of the equations (by the evenness assumption), we can drop the distinction between $\hat{\alpha}$ and $\alpha$, and between $\xi=|y|$ and $y$, so we replace terms like $\hat{\alpha}_{, \xi \xi}(|y|, t) \quad$ [cf. Eq. (8) above] by $\alpha_{, y y}(y, t)$. The resulting equations are, for $y>0$,

$$
\begin{aligned}
\dot{\alpha}^{2}+\dot{\alpha} \dot{\beta}-\alpha^{\prime \prime}-2 \alpha^{\prime 2}+\alpha^{\prime} \beta^{\prime} & =-\frac{\kappa^{2}}{3} \Lambda_{b} e^{2 \beta}, \\
-2 \ddot{\alpha}-3 \dot{\alpha}^{2}-\ddot{\beta}+2 \alpha^{\prime \prime}+3 \alpha^{\prime 2}+\beta^{\prime \prime} & =\kappa^{2} \Lambda_{b} e^{2 \beta}, \\
-\ddot{\alpha}-2 \dot{\alpha}^{2}+\dot{\alpha} \dot{\beta}+\alpha^{\prime 2}+\alpha^{\prime} \beta^{\prime} & =\frac{\kappa^{2}}{3} \Lambda_{b} e^{2 \beta},
\end{aligned}
$$

and

$$
\beta^{\prime} \dot{\alpha}+\alpha^{\prime} \dot{\beta}-\dot{\alpha}^{\prime}-\dot{\alpha} \alpha^{\prime}=0
$$

\footnotetext{
${ }^{2}$ The representation of the brane stress-energy tensor as a $\delta$-function in $y$ involves the tacit assumption that the thickness of the brane is smaller than $\left|\beta_{1}\right|^{-1}$ or $\left|\alpha_{1}\right|^{-1}$.

${ }^{3}$ The negative bulk cosmological constant ensures that one solution of the bulk equations is anti-de Sitter spacetime [1].
} 


\section{A. Power series expansion for the metric near the brane}

Equations (10)-(15) define the coupled dynamics of the brane and bulk degrees of freedom. In this section we derive a description of the dynamics of the brane by itself, by using a power series expansion for the metric near the brane.

We assume that, near $y=0$,

$$
\begin{aligned}
& \alpha(y, t)=\alpha_{0}(t)+\alpha_{1}(t)|y|+\frac{1}{2} \alpha_{2}(t) y^{2}+\cdots, \\
& \beta(y, t)=\beta_{0}(t)+\beta_{1}(t)|y|+\frac{1}{2} \beta_{2}(t) y^{2}+\cdots .
\end{aligned}
$$

This assumption is compatible with our definitions (6) and (7) of $\alpha_{1}$ and $\beta_{1}$. If we insert this expansion into Einstein's equations we obtain once again the relations (10) and (11) on the brane. Equating powers of the smooth pieces (12)-(15) of the equations of motion yields, first, from the piece of the $G_{04}$ equation (15) that is $\propto y^{0}$,

$$
\dot{\rho}+3 \dot{\alpha}_{0}(\rho+p)=0
$$

and five others that follow from the remaining terms:

$$
\begin{aligned}
& \dot{\alpha}_{0}^{2}+\dot{\beta}_{0} \dot{\alpha}_{0}-2 \alpha_{1}^{2}+\alpha_{1} \beta_{1}-\alpha_{2}=-\frac{\kappa^{2} \Lambda_{b} \exp \left(2 \beta_{0}\right)}{3} \\
& -2 \ddot{\alpha}_{0}-3 \dot{\alpha}_{0}^{2}-\ddot{\beta}_{0}+3 \alpha_{1}^{2}+2 \alpha_{2}+\beta_{2}=\kappa^{2} \Lambda_{b} \exp \left(2 \beta_{0}\right) \\
& -\dot{\alpha}_{2}+\alpha_{2}\left(\dot{\beta}_{0}-\dot{\alpha}_{0}\right)+\beta_{2} \dot{\alpha}_{0}+\beta_{1} \dot{\alpha}_{1}+\dot{\beta}_{1} \alpha_{1}-\dot{\alpha}_{1} \alpha_{1}=0 \\
& -\ddot{\alpha}_{0}-2 \dot{\alpha}_{0}^{2}+\dot{\alpha}_{0} \dot{\beta}_{0}+\alpha_{1}\left(\alpha_{1}+\beta_{1}\right)=\frac{\kappa^{2} \Lambda_{b} \exp \left(2 \beta_{0}\right)}{3} \\
& \alpha_{2}\left(2 \alpha_{1}+\beta_{1}\right)+\alpha_{1} \beta_{2}-\ddot{\alpha}_{1}-4 \dot{\alpha}_{1} \dot{\alpha}_{1}+\dot{\alpha}_{0} \dot{\beta}_{1}+\dot{\beta}_{0} \dot{\alpha}_{1} \\
& =\frac{2 \kappa^{2} \Lambda_{b} \beta_{1} \exp \left(2 \beta_{0}\right)}{3} .
\end{aligned}
$$

Equation (17) is simply the usual conservation of energy equation, and its derivation from the $G_{04}$ component of the 5D Einstein equations was already given by Binétruy et al. [5]. Using Eq. (11) in Eq. (21) we find

$$
-\ddot{\alpha}_{0}-2 \dot{\alpha}_{0}^{2}+\dot{\alpha}_{0} \dot{\beta}_{0}=\left(\frac{\kappa^{2} \Lambda_{b}}{3}+\frac{\kappa^{4} \rho(\rho+3 p)}{36}\right) \exp \left(2 \beta_{0}\right)
$$

defining a new time variable $\hat{t}$ by $d \hat{t}=\exp \left(\beta_{0}\right) d t$, we obtain the equation already found by Binétruy et al. [5] and Csaki et al. [6],

$$
-\frac{d^{2} \alpha_{0}}{d \hat{t}^{2}}-2\left(\frac{d \alpha_{0}}{d \hat{t}}\right)^{2}=\frac{\kappa^{2} \Lambda_{b}}{3}+\frac{\kappa^{4} \rho(\rho+3 p)}{36} .
$$

Note that $\hat{t}$ is just the conventional cosmological proper time, that is, the proper time as measured by comoving observers on the brane. If we assume that $\rho=\sigma+\rho_{m}$ and $p=-\sigma$ $+p_{m}$, where $\sigma$ represents the contribution from the brane tension, and $\rho_{m}$ and $p_{m}$ are the density and pressure due to the matter, then this equation reduces to

$$
\begin{aligned}
-\frac{d^{2} \alpha_{0}}{d \hat{t}^{2}}-2\left(\frac{d \alpha_{0}}{d \hat{t}}\right)^{2}= & \frac{\kappa^{2} \Lambda_{b}}{3}-\frac{\kappa^{4} \sigma^{2}}{18}+\frac{\kappa^{4} \sigma\left(3 p_{m}-\rho_{m}\right)}{36} \\
& +\frac{\kappa^{4} \rho_{m}\left(\rho_{m}+3 p_{m}\right)}{36}
\end{aligned}
$$

We shall find exact solutions of Eq. (25) below.

To complete the solution, note that Eqs. (18), (19) and (22) are algebraic equations for $\alpha_{2}$ and $\beta_{2}$. Equation (18) implies that

$$
\begin{aligned}
\alpha_{2} & =\dot{\alpha}_{0}^{2}+\dot{\alpha}_{0} \dot{\beta}_{0}-2 \alpha_{1}^{2}+\alpha_{1} \beta_{1}+\frac{\kappa^{2} \Lambda_{b} \exp \left(2 \beta_{0}\right)}{3} \\
& =\dot{\alpha}_{0}^{2}+\dot{\alpha}_{0} \dot{\beta}_{0}+\left(\frac{\kappa^{2} \Lambda_{b}}{3}-\frac{\kappa^{4} \rho(4 \rho+3 p)}{36}\right) \exp \left(2 \beta_{0}\right),
\end{aligned}
$$

where Eq. (11) was used to get the second expression. Using this result for $\alpha_{2}$ in Eq. (19) gives

$$
\begin{aligned}
\beta_{2} & =\frac{\kappa^{2} \Lambda_{b} \exp \left(2 \beta_{0}\right)}{3}+\dot{\alpha}_{0}^{2}+2 \ddot{\alpha}_{0}+\ddot{\beta}_{0}+\alpha_{1}^{2}-2 \alpha_{1} \beta_{1}-2 \dot{\alpha}_{0} \dot{\beta}_{0} \\
& =-3 \dot{\alpha}_{0}^{2}+\ddot{\beta}_{0}+\left(\frac{\kappa^{4} \rho^{2}}{12}-\frac{\kappa^{2} \Lambda_{b}}{3}\right) \exp \left(2 \beta_{0}\right)
\end{aligned}
$$

where we used Eqs. (11) and (21) in the last line. It is easy to check that once we have determined $\alpha_{2}$ and $\beta_{2}$, the remaining equations (20) and (22) are both satisfied identically. Note that the function $\beta_{0}(t)$ is not determined from this power series expansion near the brane. Thus, it is not specified by physics on the brane itself. It is determined by the dynamics in the bulk, but is gauge dependent.

\section{AN EQUATION FOR $\boldsymbol{H}^{2}$}

We now turn to the task of solving the second order dynamical equation (25) for $\alpha_{0}(t)$. The easiest way to do this is to rewrite it as a first order equation for $H^{2}=\left(d \alpha_{0} / d \hat{t}\right)^{2}$ as a function of $\alpha_{0}$. This rewriting may be accomplished by noting that

$$
\frac{d^{2} \alpha_{0}}{d \hat{t}^{2}}=\frac{d\left(d \alpha_{0} / d \hat{t}\right)}{d \alpha_{0}} \frac{d \alpha_{0}}{d \hat{t}}=H \frac{d H}{d \alpha_{0}}=\frac{1}{2} \frac{d H^{2}}{d \alpha_{0}} ;
$$




$$
\begin{aligned}
-\frac{1}{2} \frac{d H^{2}}{d \alpha_{0}}-2 H^{2}= & -\frac{\exp \left(-4 \alpha_{0}\right)}{2} \frac{d\left[H^{2} \exp \left(4 \alpha_{0}\right)\right]}{d \alpha_{0}} \\
= & \frac{\kappa^{2} \Lambda_{b}}{3}-\frac{\kappa^{4} \sigma^{2}}{18}+\frac{\kappa^{4} \sigma\left(3 p_{m}-\rho_{m}\right)}{36} \\
& +\frac{\kappa^{4} \rho_{m}\left(\rho_{m}+3 p_{m}\right)}{36}
\end{aligned}
$$

or

$$
\begin{aligned}
\frac{d\left[H^{2} \exp \left(4 \alpha_{0}\right)\right]}{d \alpha_{0}}= & \exp \left(4 \alpha_{0}\right)\left[\frac{\kappa^{4} \sigma^{2}}{9}-\frac{2 \kappa^{2} \Lambda_{b}}{3}\right. \\
& \left.+\frac{\kappa^{4} \sigma\left(\rho_{m}-3 p_{m}\right)}{18}-\frac{\kappa^{4} \rho_{m}\left(\rho_{m}+3 p_{m}\right)}{18}\right] .
\end{aligned}
$$

Note that Eq. (30) represents a linear, first-order equation for $H^{2}$, if we regard the right hand side as a source term that is (implicitly) a function of $\alpha_{0}$. Implicit in our change of variables from $t$ to $\alpha_{0}$ is the restriction to phases of the evolution of the Universe in which the two variables are related to one another monotonically. For oscillating Universes, or Universes in which the scale factor $a(t)=\exp \left[\alpha_{0}(t)\right]$ may have a contracting phase as well as one or more expanding ones, we can derive Eq. (30) for each of these phases separately.

To solve Eq. (30) generally, let us first rewrite the equation of energy conservation, Eq. (17), as

$$
\frac{d \rho_{m}}{d \alpha_{0}}+3\left(\rho_{m}+p_{m}\right)=0
$$

this equation implies

$$
p_{m}=-\rho_{m}-\frac{1}{3} \frac{d \rho_{m}}{d \alpha_{0}}
$$

Substituting this result for $p_{m}$ into equation Eq. (30) yields

$$
\begin{aligned}
\frac{d\left[H^{2} \exp \left(4 \alpha_{0}\right)\right]}{d \alpha_{0}}= & \exp \left(4 \alpha_{0}\right)\left[\frac{\kappa^{4} \sigma^{2}}{9}-\frac{2 \kappa^{2} \Lambda_{b}}{3}+\frac{\kappa^{4} \sigma}{18}\right. \\
& \left.\times\left(4 \rho_{m}+\frac{d \rho_{m}}{d \alpha_{0}}\right)+\frac{\kappa^{4}}{36}\left(4 \rho_{m}^{2}+\frac{d \rho_{m}^{2}}{d \alpha_{0}}\right)\right] \\
= & \frac{d}{d \alpha_{0}}\left[\operatorname { e x p } ( 4 \alpha _ { 0 } ) \left(\frac{\kappa^{4} \sigma^{2}}{36}-\frac{\kappa^{2} \Lambda_{b}}{6}\right.\right. \\
& \left.\left.+\frac{\kappa^{4} \sigma \rho_{m}}{18}+\frac{\kappa^{4} \rho_{m}^{2}}{36}\right)\right]
\end{aligned}
$$

we can easily read off the solution

$$
H^{2}=\frac{\kappa^{4} \sigma^{2}}{36}-\frac{\kappa^{2} \Lambda_{b}}{6}+\frac{\kappa^{4} \sigma \rho_{m}}{18}+\frac{\kappa^{4} \rho_{m}^{2}}{36}+K \exp \left(-4 \alpha_{0}\right),
$$

where $K$ is a constant of integration, and may be positive or negative. In the case of the Randall-Sundrum static solution [1], we have $\kappa^{2} \sigma^{2}=6 \Lambda_{b}, \kappa^{4} \sigma=48 \pi G$, and $\rho_{m}=K=0$, giving $H=0$ and $a(t)=\exp \left[\alpha_{0}(t)\right]=$ const. An equation similar to Eq. (34) has been derived by Kraus using a different approach [Eq. (27) of Ref. [9]]. Also a version of the first order equation (34) containing only the third and fifth terms on the right hand side was obtained in Ref. [6].

Just as in standard cosmology based on 4D general relativity, the evolution of the Universe can be reduced to the solution of an equation for $\mathrm{H}^{2}$ and an equation for energy conservation. However, the equation for $H^{2}$ has a different structure than in standard cosmology for three reasons. First, there are two terms that result from the brane tension and the negative cosmological constant in the bulk; in the RandallSundrum scenario, these terms may be chosen to cancel exactly. Second, in addition to the normal term proportional to the matter density $\rho_{m}$, there is a term that is proportional to $\rho_{m}^{2}$. This high-density "correction'" term, which is reminiscent of the small-scale deviation from Newton's law found in this scenario, becomes unimportant once $\rho_{m} \ll \sigma$. Third, there is the term $K \exp \left(-4 \alpha_{0}\right)$ that arises purely from initial conditions. This is a qualitatively new feature of the RandallSundrum scenario. In cosmology based on 4D general relativity, $H^{2}$ is completely determined by the energy density of the Universe (presuming a spatially flat model, as was done above). But in the reduction from five dimensions to four dimensions done here, we find that $H^{2}$ can be specified freely at some initial time. The additional term that results decays exactly as any relativistic matter density would (although it need not be positive).

The physical interpretation of the $K \exp \left(-4 \alpha_{0}\right)$ is as follows. The most general solution of the bulk equations of motion (12)-(15) is the black hole solution [9]

$$
d s^{2}=-w(r) d \bar{t}^{2}+w(r)^{-1} d r^{2}+r^{2} \delta_{i j} d x^{i} d x^{j},
$$

where

$$
w(r)=\frac{1}{6} \Lambda_{b} r^{2}+\frac{m}{r^{2}} .
$$

The parameter $K$ is determined by the mass parameter $m$ of the solution (35); see Eq. (27) of Ref. [9]. Positive $K$ corresponds to $m>0$ and negative $K$ to $m<0$. Although the solution (35) is static, the motion of the brane through the bulk need not respect the $\bar{t}$-time translation symmetry, and thus there is a contribution to the effective 4D energy density which varies with brane proper time [9].

In specific cosmological scenarios, the additional term can be important or negligible, depending on the magnitude of $K$. More concretely, let us imagine that the Universe begins with an early, inflationary phase of expansion, during which the energy density and pressure are dominated by an inflaton field, and that once the inflaton approaches its potential minimum, its energy dissipates into relativistic particles. For example, in one model for inflation and reheating (e.g. [12]), 


$$
\begin{aligned}
& \rho_{m}=\frac{1}{2}\left(\frac{d \phi}{d \hat{t}}\right)^{2}+V(\phi)+\rho_{r}, \\
& p_{m}=\frac{1}{2}\left(\frac{d \phi}{d \hat{t}}\right)^{2}-V(\phi)+\frac{\rho_{r}}{3},
\end{aligned}
$$

where the inflaton field $\phi$ obeys the equation

$$
\frac{d^{2} \phi}{d \hat{t}^{2}}+\left(3 H+\Gamma_{\phi}\right) \frac{d \phi}{d \hat{t}}+\frac{d V(\phi)}{d \phi}=0
$$

and the radiation energy density $\rho_{r}$ is determined from

$$
\frac{d \rho_{r}}{d \hat{t}}+4 H \rho_{r}=\Gamma_{\phi}\left(\frac{d \phi}{d \hat{t}}\right)^{2} .
$$

Here $\Gamma_{\phi}$ is a decay rate that leads to the production of relativistic particles, primarily from oscillations of the inflaton field about its minimum. It is straightforward to set up and solve these equations along with Eq. (25) numerically, and we have done so. To begin the integration, we must specify not only initial values of $\phi, d \phi / d \hat{t}$ and $\rho_{r}$, but also the starting value of $H$. Our (limited) exploration ${ }^{4}$ of numerical solutions shows that, as expected, Eq. (34) is satisfied (to the accuracy of our numerical integrations) during both the inflationary epoch and the radiation-dominated era that follows reheating, even though our solutions were based on Eq. (25) (with independent variable $\alpha_{0}$ instead of $t$ ), not Eq. (34).

Using Eq. (34) along with Eqs. (37), (38) and (39), we can gain insight into how inflation might be affected by the new features of our relationship for $H^{2}$. We assume that the Randall-Sundrum condition, $\kappa^{2} \sigma^{2} / 6 \Lambda_{b}=1$, holds. Let us first consider the effect of choosing the initial value of $H$, which translates (with suitable definition of $\alpha_{0}=0$ ) into choosing a value of $K$. To keep matters as simple as possible, let us assume that $V_{0}$, the value of $V(\phi)$ in its flat portion (where we presume the inflaton starts), is smaller than $\sigma$, so we can neglect the nonlinear term in Eq. (34) for $H^{2}$. Then if the initial value of $K$ is positive and $\sim 8 \pi G V_{0} / 3$, the starting value of $H^{2}$ in inflation theory based on 4D general relativity, deviations in $H^{2}$ from its standard cosmological value will damp away exponentially as the inflaton rolls toward its minimum. Given enough expansion, $K \exp \left(-4 \alpha_{0}\right)$ becomes negligible compared with $8 \pi G V_{0} / 3$ by the end of inflation, and reheating results in a radiation density that dominates the $K \exp \left(-4 \alpha_{0}\right)$ term in Eq. (34) thereafter. If the initial value of $H$ is far larger than in standard cosmology, the inflaton

\footnotetext{
${ }^{4} \mathrm{We}$ chose $d \phi / d \hat{t}=0$ and $\rho_{r}=0$ initially in all of our numerical integrations, and also focussed on cases where the RandallSundrum condition, $\kappa^{2} \sigma^{2} / 6 \Lambda_{b}=1$ is satisfied. A simple effective potential of the form $V(\phi)=V_{0}\left[\exp (-\epsilon)-\exp \left(-\sqrt{\epsilon^{2}+\phi^{2} / \phi_{0}^{2}}\right)\right]$ was assumed, with $\epsilon$ chosen to be small but nonzero to guard against pathologies in $d V(\phi) / d \phi$ near $\phi=0$. Although $V_{0} / \sigma$ is a parameter that can be chosen arbitrarily, so far we have considered $V_{0} / \sigma \leqslant 1$.
}

hardly rolls at all until the universe expands enough that $K \exp \left(-4 \alpha_{0}\right)$ becomes comparable to $8 \pi G V_{0} / 3$; the subsequent evolution is identical to what would transpire if we had started with $H^{2} \sim 8 \pi G V_{0} / 3$. This scenario can be thought of as including a pre-inflation, "radiation" dominated era, followed by conventional inflation and reheating. If the initial value of $H$ is well below its standard value initially, which means that $K<0$ (but not so small that $H^{2}<0$ ), then the total number of e-foldings during inflation is diminished, but can still be large enough that $K \exp \left(-4 \alpha_{0}\right)$ becomes unimportant by the time reheating occurs. Excluding negative $H^{2}$ amounts to limiting $-K=|K|$ to values smaller than $\kappa^{4} V_{0}\left(\sigma+V_{0} / 2\right) / 18$ initially (at some small but nonzero starting value of $a(t)$; spatially flat models with $K<0$ have no big bang). This might not be unnatural, as we might expect $V_{0} \sim \sigma \sim|K| / \kappa^{4} \sigma$.

The nonlinear term in Eq. (34) can be important if $V_{0}$ $\gtrsim \sigma$. In that case, the Universe expands faster than in standard cosmology, but $H$ is still time-independent while $\phi$ is rolling down the flat part of $V(\phi)$, so much of the standard picture of inflation can be carried over intact. In particular, as discussed in the previous paragraph, it is possible that the Universe expands enough during inflation to render $K \exp \left(-4 \alpha_{0}\right)$ negligible before reheating. The number of e-foldings of $a(\hat{t})$ between the time when the presently observable portion of the Universe crossed the horizon and the end of inflation is $N_{H} \sim H T_{0} / V_{0}^{1 / 4} H_{0}$, where $T_{0}$ and $H_{0}$ are the present temperature and Hubble parameter. Since $N_{H}$ $\propto H$, larger $H$ means more e-foldings, and, generically, larger primordial density fluctuations, which could be problematic. When reheating is complete, it is still possible that $\rho_{r}>\sigma$ for awhile, until expansion can reverse the inequality. During the phase in which $H^{2} \approx \kappa^{4} \rho_{m}^{2} / 36$, the scale factor behaves as $a(t)=\exp \left[\alpha_{0}(t)\right] \sim t^{1 / 4}$ as found in Refs. [5,6], but once $H^{2}$ $\rightarrow \kappa^{4} \sigma \rho_{m} / 18$, the scale factor behaves as $a(t) \sim t^{1 / 2}$. As long as $\sigma$ is large compared with $\sim(\mathrm{MeV})^{4}$, the nonlinear term in Eq. (34) has no effect on cosmological nucleosynthesis.

In the scenario outlined above, inflation plays a central role in guaranteeing that the evolution of the universe tends toward the standard cosmological model by the nucleosynthesis epoch, at $T \sim 100 \mathrm{keV}-\mathrm{a}$ few $\mathrm{MeV}$. It is not sufficient to require that $a(t) \rightarrow t^{1 / 2}$ at late times to obtain agreement with standard model predictions for the synthesis of the light elements, since this solution is obtained for $K \neq 0$, even if $K \exp \left(-4 \alpha_{0}\right)>\kappa^{4} \sigma V_{0} / 18$, and $H^{2} \approx K \exp \left(-4 \alpha_{0}\right)$. The outcome of cosmological nucleosynthesis depends on the competition between $H$ and various particle reaction rates, such as those that create and destroy protons and neutrons; these reaction rates can be computed as functions of $T$ and the baryon density of the Universe [7,10]. The comparison of the observed light element abundances with the theoretical calculations constrains the ratio of $\rho_{m}$ to its standard model value at $T \sim 100 \mathrm{keV}-1 \mathrm{MeV}$, which includes contributions from photons, three neutrino flavors, and, at $T \gtrsim m_{e}$, electrons and positrons to be very close to one [10]. Thus, there is little room for additional relativistic species, such as $K \exp \left(-4 \alpha_{0}\right)$ for positive $K$. If $K>0$, it is crucial that $K /(a(t) T)^{4}$ become negligible well before nucleosynthesis 
occurs, as it is (virtually) unaffected by expansion alone. If $K \exp \left(-4 \alpha_{0}\right)<0$, it would have to be balanced nearly exactly by additional particles that are relativistic during the nucleosynthesis era. While one cannot rule out such a perverse conspiracy a priori, it would require fine tuning that seems rather unlikely, so even for negative $K$ it seems that, generically, $K /(a(t) T)^{4}$ must be negligible at $T \sim 100 \mathrm{keV}-1$ $\mathrm{MeV}$. Inflation and reheating provide a natural mechanism for decreasing $K /(a(t) T)^{4}$ to an acceptable level. Indeed, one might say that, if the Randall-Sundrum brane world scenario proves correct, inflation is required to preserve the success of the standard big bang cosmology in accounting for the abundances of the light chemical elements.

\section{CONCLUSIONS AND REMARKS}

We have re-examined the evolution of homogeneous, isotropic cosmologies in the Randall-Sundrum brane world scenario, building on the earlier work of Binétruy et al. [5] and Csaki et al. [6]. We derived the explicit form of the equations that governed the dynamics of both the bulk and brane degrees of freedom [Eqs. (10), (11), (17) and (12)-(15) above]. We derived a generalization [Eq. (34) above] of the standard relation between the Hubble parameter $H$ and energy density $\rho_{m}$. This equation contains a term (an effective 4D energy density) that acts like a free, relativistic species, and is the only effect of the 5D gravitational degrees of freedom on the bulk dynamics.

The cosmological equation we find for $H$ contains extra terms compared to $4 \mathrm{D}$ general relativity (a term proportional to density squared and the term due to $5 \mathrm{D}$ gravitational degrees of freedom) but, generically, these become unimportant at late times. In particular, we analyzed how reheating at the end of inflation smoothly matches onto the standard behavior $a(t) \propto t^{1 / 2}$ of the radiation dominated epoch, and not the behavior $a(t) \propto t^{1 / 4}$ found in Ref. [5]. We show that in- flation can drive the expansion rate of the Universe toward its standard cosmological value, $H^{2}=8 \pi G \rho_{m} / 3$, after reheating under a variety of conditions. Thus, the RandallSundrum scenario is compatible with standard cosmology.

A recent paper by Shiromizu, Maeda and Sasaki [13] derives effective Einstein equations on the brane for a general bulk metric. Their independent analysis is compatible with our results; they find corrections to the Einstein equation that scale as density squared, and also a correction term $E_{\mu \nu}$ due both to bulk gravitational waves and to non-radiative bulk degrees of freedom. Bulk gravitational waves do not arise in our analysis because of the symmetries we assumed in the starting metric (1). On the other hand, our term $K \exp \left[-4 \alpha_{0}(t)\right]$ in Eq. (34) corresponds to the non-radiative piece of the tensor $E_{\mu \nu}$ of Eq. (17) of Ref. [13].

Finally, we remark that to solve the hierarchy problem, it was suggested [14] that we live in a probe brane (or " $\mathrm{TeV}$ " brane), which is at a distance $y_{0}$ away from the Planck brane, where the graviton is trapped. In this case, the tension of the " $\mathrm{TeV}$ " brane is substantially smaller than that of the Planck brane. In the cosmological setting, depending on the details, the $\rho_{m}^{2}$ term contribution to $H^{2}$ may no longer be negligible on the " $\mathrm{TeV}$ " brane, and the standard cosmology will be modified accordingly. It will be interesting to see if cosmological constraints can be consistent with this solution to the hierarchy problem.

\section{ACKNOWLEDGMENTS}

We thank Gary Shiu for helpful discussions. We are grateful to P. Kraus for pointing out an error in interpretation in an earlier version of this paper. É.F. was supported in part by NSF grant PHY 9722189 and by the Alfred P. Sloan foundation. The research of S.-H.H.T. is partially supported by the National Science Foundation. I.W. gratefully acknowledges support from NASA.
[1] L. Randall and R. Sundrum, Phys. Rev. Lett. 83, 3370 (1999); 83, 4690 (1999); M. Gogberashvili, hep-ph/9812296; Europhys. Lett. 49, 396 (2000); Mod. Phys. Lett. A 14, 2025 (1999).

[2] N. Arkani-Hamed, S. Dimopoulos, and G. Dvali, Phys. Lett. B 429, 263 (1998).

[3] N. Kaloper, Phys. Rev. D 60, 123506 (1999); T. Nihei, Phys. Lett. B 465, 81 (1999).

[4] P. J. Steinhardt, Phys. Lett. B 462, 41 (1999); H. B. Kim and H. D. Kim, Phys. Rev. D 61, 064003 (2000); L. Mersini, hep-ph/9909494; O. DeWolfe, D. Z. Freedman, S. S. Gubser, and A. Karch, Phys. Rev. D (to be published), hep-th/9909134; P. Kanti, I. I. Kogan, K. A. Olvie, and M. Pospelov, Phys. Lett. B 468, 31 (1999); J. M. Cline, C. Grojean, and G. Servant, Phys. Rev. Lett. 83, 4245 (1999).

[5] P. Binétruy, C. Deffayet, and D. Langlois, Nucl. Phys. B565, 269 (2000).

[6] C. Csaki, M. Graesser, C. Kolda, and J. Terning, Phys. Lett. B 462, 34 (1999).

[7] See, e.g., P. J. E. Peebles, Principles of Physical Cosmology (Princeton University Press, Princeton, NJ, 1993), pp. 184-
196; E. W. Kolb and M. S. Turner, The Early Universe (Addison-Wesley, New York, 1990), pp. 87-111; or B. E. J. Pagel, Nucleosynthesis and the Chemical Evolution of Galaxies (Cambridge University Press, Cambridge, England, 1997), pp. 103-130.

[8] H. J. Schmidt, Int. J. Mod. Phys. D 7, 215 (1998).

[9] P. Kraus, J. High Energy Phys. 12, 011 (1999).

[10] See, for example, D. N. Schramm, The Light Element Abundances, edited by P. Crane (Springer-Verlag, Berlin, 1995), p. 51 .

[11] A. Guth, Phys. Rev. D 23, 347 (1981); A. D. Linde, Phys. Lett. 108B, 389 (1982); A. D. Linde, ibid. 129B, 177 (1983); A. Albrecht and P. J. Steinhardt, Phys. Rev. Lett. 48, 1220 (1982).

[12] A. Albrecht, P. J. Steinhardt, M. S. Turner, and F. Wilczek, Phys. Rev. Lett. 48, 1437 (1982); L. Abbott, E. Farhi, and M. Wise, Phys. Lett. 117B, 29 (1982); A. Dolgov and A. D. Linde, ibid. 116B, 329 (1982).

[13] T. Shiromizu, K. Maeda, and M. Sasaki, Phys. Rev. D 62, 024012 (2000).

[14] J. Lykken and L. Randall, hep-th/9908076. 